\title{
Applicability of Transactional Memory to Modern Codes
}

B. L. Bihari

July 26,2010

International Conference on Numerical Analysis and Applied Mathematics Rhodes, Greece September 19, 2010 through September 25, 2010 
This document was prepared as an account of work sponsored by an agency of the United States government. Neither the United States government nor Lawrence Livermore National Security, LLC, nor any of their employees makes any warranty, expressed or implied, or assumes any legal liability or responsibility for the accuracy, completeness, or usefulness of any information, apparatus, product, or process disclosed, or represents that its use would not infringe privately owned rights. Reference herein to any specific commercial product, process, or service by trade name, trademark, manufacturer, or otherwise does not necessarily constitute or imply its endorsement, recommendation, or favoring by the United States government or Lawrence Livermore National Security, LLC. The views and opinions of authors expressed herein do not necessarily state or reflect those of the United States government or Lawrence Livermore National Security, LLC, and shall not be used for advertising or product endorsement purposes. 


\title{
Applicability of Transactional Memory to Modern Codes
}

\author{
Barna L. Bihari \\ Lawrence Livermore National Laboratory, 7000 East Avenue, Livermore, CA 94550, USA
}

\begin{abstract}
In this paper we illustrate the features and study the applicability of transactional memory (TM) as an efficient and easy-to-use alternative for handling memory conflicts in multi-theaded physics simulations that use shared memory. The tool used for our preliminary analysis of this novel construct is IBM's freely available Software Transactional Memory (STM) system. Instead of attempting to apply it to a production grade simulation code, we developed a much simpler test code that exhibits most of the salient features of modern unstructured mesh algorithms, but without the complicated physical models. We apply STM to two frequently used algorithms in realistic multi-physics codes. Our computational experiments indicate a good fit between these application scenarios and the TM features.
\end{abstract}

Keywords: Algorithms, Transactional Memory, Finite Volume, Monte Carlo

PACS: $02,07,89.20$

\section{INTRODUCTION}

Large scale multi-physics simulations are now routinely run in addition to, or, on occasion, in lieu of actual experiments and theoretical estimates. Modern computers facilitating these computations are invariably massively parallel in terms of computer nodes, with each node being multi- (or "many-") core which all share the same memory address space. On the node-level, no explicit domain decomposition and message passing needs to take place, so this locally parallel model appears to be easy to program via threads. However, if not done carefully, threaded code can have insidious bugs and dramatic inefficiencies, mainly due to race conditions that cause memory conflicts and ultimately give the wrong answer which is not even reproducible.

Avoiding memory conflicts by clever programming can become an art or an impossible task, depending on the circumstances governed by the physics and the algorithms that simulate it. Barriers and mutex type locks tend to degrade or altogether destroy parallel performance and can even result in dead-locks. As an alternative, transactional memory (TM) has received considerable attention lately as a construct that is both convenient and efficient for handling memory contention. It offers two of the four "ACID" (i.e. atomicity, consistency, isolation, durability) properties: atomicity and isolation. For a background on TM, see [1], for example. In our study we use a version of IBM's software transactional memory (STM) compiler that implements weak isolation meaning that variables are only safe if they are accessed within transactions and not from other parts of the code (see [2].)

The underlying idea that sets TM apart from other synchronization techniques is that it does not block threads when a memory conflict is likely to occur. Instead, TM treats memory accesses and updates as transactions which are executed in isolation without being held up by locks, but which are checked afterwards for memory violations. In such cases, the system is able to unroll a transaction resulting in a retry of the same memory operation. The retries are expected to be potentially costly, but since conflicts are assumed to be few in number, the overall cost should still be substantially lower than blocking every thread.

As with all new software, it takes some effort to install, tune, learn and apply it. STM is no exception. In addition, since the present tool is indeed a software implementation, it has relatively high overhead even without any conflicts. At the current stage of experimentation, we are merely testing its applicability to a few frequently used numerical methods. There will be some for which it may be the perfect fit, and others for which its benefits may be limited. In the rest of this paper we briefly describe two algorithms which appear to greatly benefit from TM. For the time being, our main goal is to provide some insight into the applicability of this exotic construct in terms of conflict management and without much emphasis on the wall clock time. However, as our results in [4] show, in some cases even STM appears to outperform its more traditional counterpart, the OpenMP "critical" statement. 


\section{THE ALGORITHMS AND TEST CODE}

One particular area where the transactional memory paradigm may provide great benefits is unstructured-mesh multiphysics simulation codes which have been gaining in popularity because of their geometric and architectural flexibilty. Such codes typically use numerous compute-intensive loops, are fairly scalable when domain-decomposed for MPI communication, and tend to have complicated memory-referencing patterns. Adding OpenMP to this mix is a challenge that, to date, few developers have attempted to tackle. A major obstacle presented by the injection of shared-memory parallelism into an already complicated code structure is, of course, the presence of potential memory conflicts.

Transactional memory, on the other hand, promises to provide most, if not all, of the attributes of thread-safety but with much reduced barrier and lock times. While pure hardware implementations are sparse or nonexistent, software transational memory allows one to experiment with the applicability of TM in a particular code environment. We will present some results using IBM's STM compiler with a new test code called BUSTM (Benchmark for Unstructuredmesh Software Transactional Memory) that will likely evolve into a full-fledged benchmark. Such test codes are simple enough to provide for a clear understanding of the programming issues, but still reproduce the algorithms and mimic the behavior of their real-life counterparts.

In constructing such a code, one needs to make sure that the salient features of the target code are reproduced by the benchmark. Race conditions being our main focus here, we artifically generate memory conflicts in either a deterministic or random yet still controllable manner. That is, even when the conflicts are random, their probabilities are indirectly configurable by appropriate run parameters. Another requirement for the code is rigorous error-checking by designing examples where the answer is computable independently of the threaded transactional memory experiments.

The BUSTM code, as it's name would suggest, aims to use realistic unstructured mesh connectivity. The bookkeeping of such codes tends to be fairly complex yet flexible. Our code, for example, can handle unstructured cells with an arbitrary number of faces; specific cell types used to date include triangular prism, hexahedron, tetrahedron and pyramid. The basic building blocks of nodes, faces and cells are cross-referenced in almost all combinations, so that indirect indexing is pervasive throughout the code. As such, this also provides what intuitively seems like a very fertile ground for memory conflict resolution via transactional memory. We test this conjecture on both deterministic and probabilistic conflicts on one particular mesh, with more examples to follow in the final paper.

\section{COMPUTATIONAL EXPERIMENTS}

\section{Deterministic Conflicts}

Conservative finite volume schemes on unstructured grids can and have been successfully parallelized via domain decomposition and MPI message passing in a distributed memory framework. Careful evaluation of the memory access patterns, however, reveals that as the face-based loops are traversed, conflicts may occur as the cells are updated. The probability of such conflicts is extremely low, but cannot be ruled out, and, if ignored, can lead to (slightly) incorrect execution and turn into a debugging nightmare. This is therefore a case where traditional thread-safety with locks and barrier can completely ruin performance and where transactional memory may have a huge potential payoff.

Short of an actual CFD solver, we "simulate" face-by-face flux computations by computing the numerical divergence of a mesh-function $F$ that is defined on an unstructured mesh in a cell-centered sense. This involves computing the gradient of a function. If the function is constant, it's gradient (and therefore divergence) will be zero. The relevant code section in the called routine is brief and simple, and can be seen in [3], along with the calling sequence.

Our illustrative example is a small mesh of 3000 hexahedral cells arranged as a 2-D layer of 3-D cells. There were 12110 quadrilateral faces and 6324 nodes. The number of (resolved) conflicts comes directly from the statistical output available as a run-time option to the IBM STM compiler. As we plot this for each of the 1000 runs performed in order to get some basic statistical sampling (see Fig. 1(a)), it is clear that the number of conflicts is very small or zero during each particular run (less than 20) and they seem random within that range.

The total number of resolved conflicts by STM along with the total number of errors committed (without STM) during all 1000 runs are depicted on Fig. 1(b). This figure illustrates that conflicts did not occur on (one or) two threads during any of the 1000 runs. It is also obvious that generally the number of conflicts, as well as errors, increases as the number of threads increases, but not necessarily in a linear fashion.

The number of STM-retries (ie. rollbacks) is comparable to the number of erroneous results, but both are extremely small compared to the number of potential conflicts. In fact, by today's standards, this is a very small mesh, but even 
on 16 threads the conflict occurrence was at most $0.1 \%$ when measured against the total number of cell updates. We also note that despite of the difference between these two numbers, they both show similar trends as the number of threads increase. As such, they can serve as a sanity check for the STM statistics output, as well as for the benchmark's error checking mechanism.
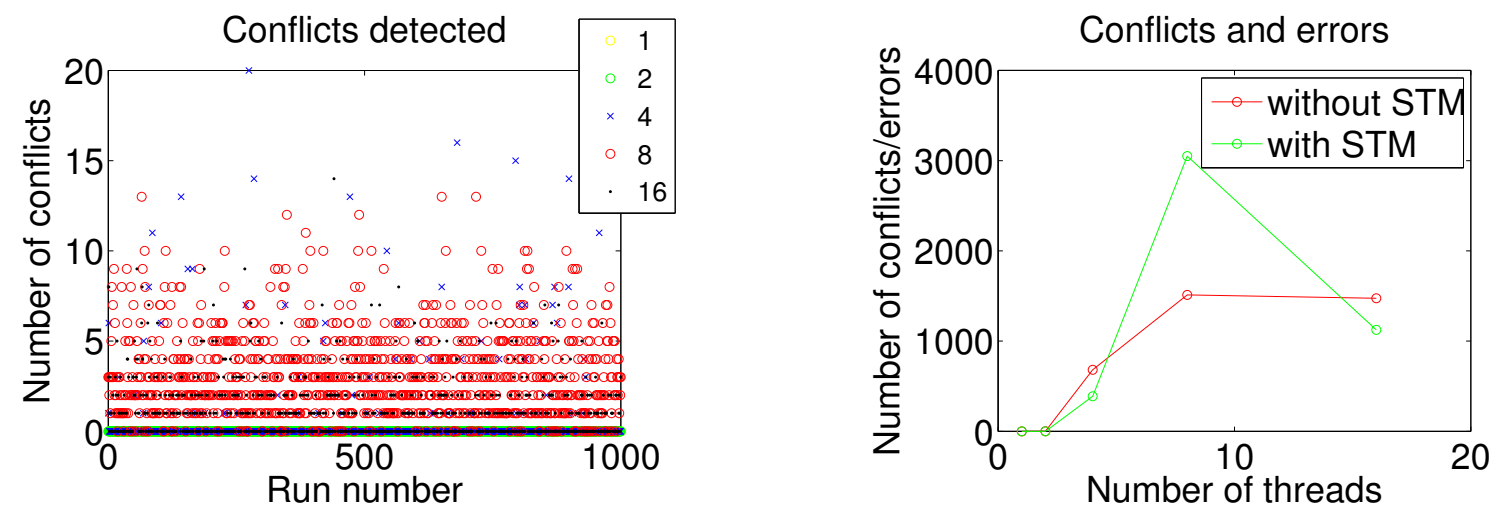

FIGURE 1. Deterministic conflicts on hex mesh: (a) for each run and number of threads; (b) total conflicts and errors

\section{Probabilistic Conflicts}

Another common occurrence of potential memory conflicts is in threaded implementations of Monte Carlo codes where randomly released particles travel through a computational mesh and increment a cell-based physical quantity each time they touch a cell. Parallelizing over the total number of particles leads to almost embarassingly parallel loops, except for the race condition produced by two particles (belonging to two different threads) trying to update the same cell at the same time. As in the deterministic case, the chances of a memory conflict occuring is extremely low, but given sufficient number of particles and threads, it will eventually happen.

To simulate the behavior of particles without having an actual Monte Carlo code we take advantage of the wealth of information given to us by the unstructured bookkeeping used in BUSTM. Instead of having the particles travel along a straight line through space, they travel from cell to cell via the neighbor information available for each adjacent cell. Therefore, there are actually two levels of randomness injected into the code:

- (i) the cell in which the particle is "born" is randomly selected, and

- (ii) the cell-neighbor that is next on the particle's path is randomly chosen as well.

In order to make the code highly configurable, the number of particles is independent of the number of cells. After being created, or "born," each particle is "alive" as long it stays withing the computational domain, ie. the face through which it exits the current cell is an interior face. If that face (again, randomly selected) is a boundary face, it exits the domain and it's path is completed. One quickly realizes that there will be a huge variability in the particle path lenghts, with some having a short life-span, while others staying active within the domain for a long time. This is indeed a hallmark of Monte Carlo simulations, and it can cause poor parallel scalability of such codes.

The atomic region required to make this algorithm thread-safe is again trivially brief. We chose to increment a single cell-based integer counter within the transaction; for details see again [3].

The calling routine essentially distributes the particles to each thread where each particle, once randomly generated, is guided through the mesh by the given connectivity until it exits the domain. A separate counter is incremented for each particle, so that the total number of touches needs to be cell_counter*particle_counter which provides for an independent error checking mechanism. Without using TM, we typically get the wrong number of total touches.

For our experiements, we again used our hexahedral mesh of the previous section. As before, the number of rollbacks comes from the statistical output of the STM compiler. We again performed 1000 runs, using 15000 random particles ( 5 times of the number of cells) during each run. The results from Fig. 2(a) now show a much higher number of conflicts than in the deterministic case. The conflicts are fairly evenly distributed in each run, and their number also seems to be almost linearly proportional to the number of threads used. Certainly, this pattern is clearly visible only because there are sufficient number of conflicts for a statistical sampling. 

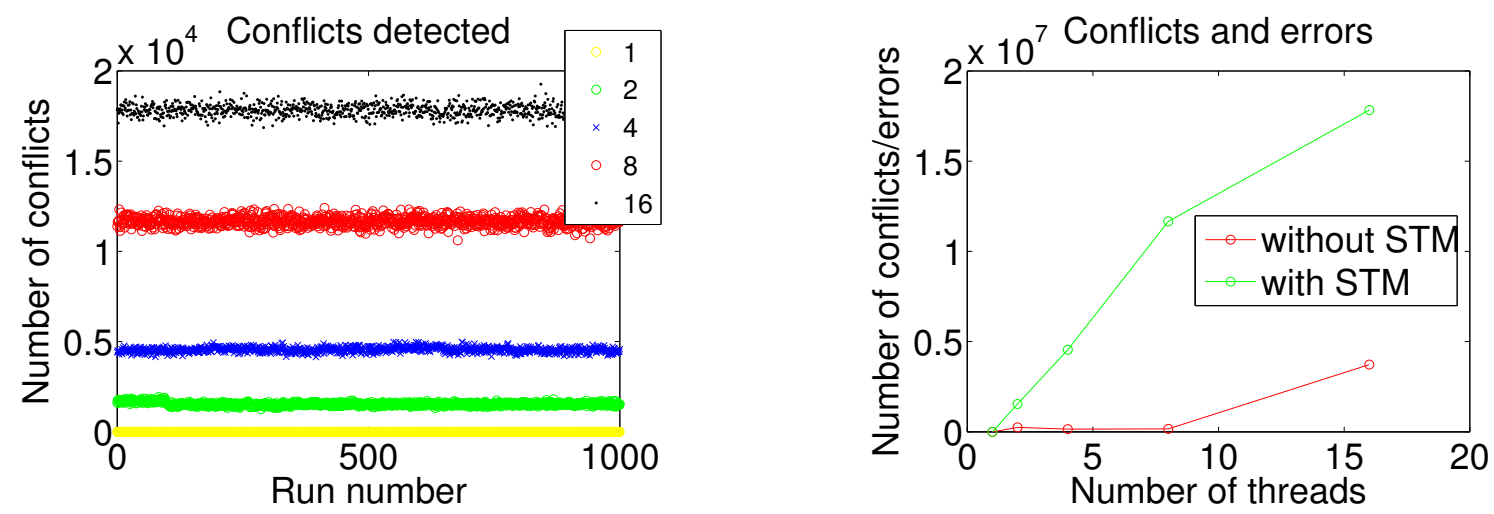

FIGURE 2. Probabilistic conflicts on hex mesh: (a) for each run and number of threads; (b) total conflicts and error

Interestingly, when one compares the difference between total number of resolved conflicts by STM and the total number of errors committed (without STM) (see Fig. 2(b), the trend is opposite of what it was in the deterministic case. We find that STM overpredicts in all cases, sometimes by an order of magnitude. While more experimentation is necessary, this may be due to the much heavier computational load imposed by the frequent invocation of a random number generator. The total number of both the resolved conflicts and committed errors is still small compared to the number of updates. For example, on 16 threads the conflict probability went up to $0.26 \%$ on this relavitely small mesh.

\section{DISCUSSION}

While the deterministic and probabilistic algorithms are very different in terms of the numerical algorithms they intend to represent, and strikingly different in the resolved conflicts vs. errors comparison, they have some similarities as well. In both cases we end up with very small conflict probabilities and both work on the same unstructured mesh.

Indeed, they both appear to be the perfect cases where very few conflicts occur, so if the price to be paid for their resolution were proportional to their number, it would still be relatively small overall cost. Achieving this same result with mutex-type locks, on the other hand, would be prohibitively expensive. While the overhead incurred by the software implementation of TM prevents us from accurate time measurements of the roll-backs, these sort of experiments allow for algorithm analysis in preparation (and hope) for an eventual hardware implementation that is much more efficient. Preliminary timing results comparing OpenMP critical and atomic suggest that, surprisingly, even with the known large overhead of STM, TM is already slightly faster (overall about 10\%) than the corresponding lock-based implementation (see [4].)

\section{ACKNOWLEDGMENTS}

This work was performed under the auspices of the U.S. Department of Energy by Lawrence Livermore National Laboratory under Contract DE-AC52-07NA27344.

\section{REFERENCES}

1. J. Larus, and C. Kozyrakis, Communications of the ACM 51, 80-88, (2008).

2. IBM, "IBM XL C/C++ for Transactional Memory for AIX, v0.9 Language Extensions and Users Guide," URL http://dl.alphaworks.ibm.com/technologies/xlcstm/xlcstm-whitepaper.pdf, May 2008.

3. M. Wong, B. L. Bihari, B. R.de Supinski, P. Wu, M. Michael, Y. Liu, W. Chen, IWOMP 2010 Conference Proceedings LNCS 6132, 149-160, (2010).

4. B. L. Bihari, "Experiments Using IBM's Software Transactional Memory Compiler" in ScicomP 15 Meeting, URL http://spscicomp.org/ScicomP15/slides/user/bihari.pdf, Barcelona, Spain, May 2009. 